\title{
Sensor Placement for Real-Time Dynamic State Estimation in Power Systems: Structural Systems Approach
}

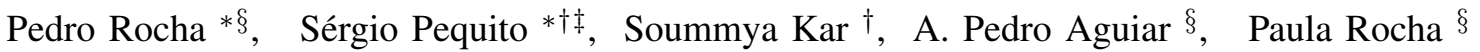 \\ * The first two authors contributed equally to this work. \\ $\dagger$ Department of Electrical and Computer Engineering, Carnegie Mellon University, Pittsburgh, PA 15213 \\ (e-mail: spequito@andrew.cmu.edu, soummyak@andrew.cmu.edu) \\ ${ }^{\ddagger}$ Institute for Systems and Robotics, Instituto Superior Técnico, Technical University of Lisbon, Lisbon, Portugal \\ $\S$ Department of Electrical and Computer Engineering, Faculty of Engineering, University of Porto, Porto, Portugal \\ (e-mail: sabino326@gmail.com, pedro.aguiar@fe.up.pt, mprocha@fe.up.pt)
}

\begin{abstract}
This paper studies the problem of sensor placement design for efficient dynamic real-time state estimation in electric power networks. Given a (linearized) dynamic physical model of the power system, efficient sensor placement strategies are proposed that minimize the observability index of the system. The observability index plays a key role in determining the minimum window length of filters that guarantee stable estimation error and minimizing this index allows the design of memory and computationally efficient filtering schemes with performance guarantees. Specifically, given the system dynamics, the paper addresses the following two sensor placement design problems: (1) determining the minimal number and placement of sensors that achieves a certain desired system observability index, and (2) given the number of sensors to be deployed, obtaining the placement achieving minimal system observability index. These problems are addressed in a structural systems framework, i.e., the placement strategies are obtained on the basis of the sparsity pattern (location of zeroes/non-zeroes) of the system coupling matrix, and the design guarantees hold for almost all numerical parametric realizations of the system. Finally, an example is provided which illustrates the analytical findings.
\end{abstract}

Index Terms-Structural Systems, Dynamic Observability, Real-Time Estimation

\section{INTRODUCTION}

In the last decade, special attention has been given to synchronized phasor measurements, which grasp the potential to enhance the monitoring of the power grid. Thus, enabling near real-time system control and dynamic state estimation, see [4] and references therein. However, the high sampling frequency of the different technologies put a huge strain on the communication and data processing infrastructure of the grid. This motivates the study of judicious sensor placement to obtain the minimum number of sensors and the corresponding placement locations that guarantee system observability. The judicious placement of phasor measurement units (PMUs) technology designed to accurately observe key power system metrics - has been studied, for instance, in [11], [19] and references therein. Nevertheless, state-of-the-art techniques in sensor and/or PMU placement typically consider static observability as the placement criterion or metric of interest, which is fundamentally and conceptually different from our current goal of ensuring dynamic observability, see [4] for a study on their implications in state monitoring of the power electric grid. Ensuring dynamic observability is, in fact, a fundamental system-theoretic requirement for designing stable and meaningful system estimators/observers.

Our approach, rather than focusing on a specific large-scale system with a specified set of (numerical) parameter values, is hinged on structural systems theory (see [3] for a survey), which allows for a unified design and analysis treatment of equivalence classes of such systems. For instance, in the context of linear time-invariant (LTI) systems, the equivalence classes described above are given by the sparsity pattern of the state-space matrices of the system; as such, structural system approaches may be used to provide a unified design criteria for all the systems in an equivalence class, i.e., with the same sparsity. Clearly, as a by-product, such an approach often leads to robust design procedures, especially in systems with uncertain or unknown parameter values, as is commonly the case in power electric grids. To be precise, design and analytical certificates formulated and obtained through structural systems theory hold for almost all parameter values, except on a manifold of zero Lebesgue measure, see [13]. When most of the systems in an equivalence class are dynamically observable, we say that the system is structurally observable (see the next section for formal definition).

In practice, we are also interested in understanding how fast the initial state vector can be recovered for a given system, hence enabling real-time monitoring. Additionally, the implementation of estimators to retrieve the initial state of the system, as well as mandatory policy laws, commonly put a strain on storage, which motivated us to look for alternative tools to address sensor placement. In fact, inspired by the implications of the observability index [2] in the performance of state estimators for dynamical systems, hereafter we focus in its structural counterpart, the structural observability index, that was introduced in [6], and computational methods for its computations were proposed in [15]. Further, until recently, only upper bounds of the structural observability index were known and given in terms of graph representations [17]. However, there is a lack of methods concerning system design when restrictions are imposed on the systems' structural observability index. Recently, it was shown that the problem of obtaining the minimum number of state variables to ensure a specified structural observability index is NP-hard [14].

As emphasized earlier, the main drawback of static observability based formulations is that they do not take into account 
the system dynamics; as such, the resulting placement configurations do not generally guarantee desired dynamic system observability properties, the fundamental requirement for the design of dynamic state estimators with error stability. The literature on sensor placement design in power electric grids to ensure dynamical observability is scarce, see for instance [10] and references therein. In general, sensor placement strategies aim to optimize some function of the observability Grammian, which is usually hard [7]. Subsequently, some heuristics were proposed in [12], whereas greedy algorithms with optimality guarantees are provided in [16], [18]. Nevertheless, most of these approaches assume that the model with accurate parameters is given, which is not commonly true when dealing with power electric grids. Alternatively, structural systems observability analysis and sensor placement has been previously addressed, for example in [3] and references therein. The present work is motivated by the results published recently in [9] and [8], where optimal sensor placement design to ensure structural observability is characterized, together with efficient (polynomial in the number of dynamical state variables) algorithms.

The main contributions of the present paper consist in providing a greedy algorithm to obtain the solution to the following two problems: (1) determining the minimal number and placement of sensors that achieve a certain desired system observability index, and (2) given the number of sensors to be deployed, obtaining the placement that ensures that the system observability index is minimal. Additionally, we explore the solution to the above problems when the dynamic system models a power electric grid as proposed in [5].

The rest of this paper is organized as follows. In Section III we formally present the problems addressed in this paper. Section II introduces some preliminary concepts and results in structural systems theory to be used in the sequel. Section IV presents the main results of this paper. In Section V, we briefly review the dynamical model of the power electric grid modeled as in [5]. Finally, an illustrative example on the IEEE 5-bus system (with 18 dynamical state variables) is provided in Section VI.

\section{Preliminaries And Terminology}

Consider a (possible large-scale) linear-time invariant (LTI) system

$$
x_{k+1}=A x_{k}, \quad y_{k}=C x_{k},
$$

where $x \in \mathbb{R}^{n}$ and $y \in \mathbb{R}^{m}$ are the system state and measured output, respectively. Let us define $\mathcal{O}^{k}(A, C)$ as the $k$-observability matrix associated with the pair $(A, C)$ as $\mathcal{O}^{k}(A, C)=\left[C^{T}(C A)^{T} \cdots\left(C A^{k-1}\right)^{T}\right]^{T}$. For dynamical systems of the form (1), the observability index associated with the pair $(A, C)$, given by

$$
\mu(A, C)=\min \left\{\left\{k \in \mathbb{N}: \operatorname{rank}\left[\mathcal{O}^{k}(A, C)\right]=n\right\}, \infty\right\},
$$

counts the number of iterations, at which the measurements of the system are collected, that one has to wait until it is possible to recover the (unknown) initial condition $x_{0}$. The pair $(A, C)$ is observable if and only if $\mu(A, C)<\infty$.
Suppose that the sparsity (i.e., location of zeroes and nonzeroes) pattern of $A$ is available, but the specific numerical values of its non-zero elements are not known. Let $\bar{A} \in\{0,1\}^{n \times n}$ be the binary matrix that represents the structural pattern of $A$, i.e., it encodes the sparsity pattern of $A$ by assigning 0 to each zero entry of $A$ and 1 otherwise. A pair $(A, C)$ is said to be structurally observable if and only if almost all pairs $\left(A^{\prime}, C^{\prime}\right)$ with the same structure as $(A, C)$, i.e., same locations of zeroes and non-zeroes, are observable. Now, we introduce the structural counterpart of the observability index, to be referred to as structural observability index or generic observability index and given by

$$
\mu_{G}(\bar{A}, \bar{C})=\min \left\{\left\{k \in \mathbb{N}: \max _{\substack{A \in[\bar{A}] \\ C \in[\bar{C}]}} \operatorname{rank}\left[\mathcal{O}^{k}(A, C)\right]=n\right\}, \infty\right\},
$$

where the equivalence class $[\bar{M}]=\left\{M \in \mathbb{R}^{n \times m}: M_{i, j}=\right.$ 0 if $\left.\bar{M}_{i, j}=0\right\}$ is induced by $\bar{M} \in\{0,1\}^{n \times m}$. Therefore, the structural pair $(\bar{A}, \bar{C})$ is structurally observable if and only if $\mu_{G}(\bar{A}, \bar{C})<\infty$. Further, if a structural pair $(\bar{A}, \bar{C})$ has a generic observability index $\mu_{G}(\bar{A}, \bar{C})=p$, then almost all pairs $(A, C)$ with the same sparseness of $(\bar{A}, \bar{C})$ have a observability index $p$, except on a manifold with zero Lebesgue measure.

Given a dynamical system (1), an efficient approach to the analysis of its structural properties is to associate it with a directed graph (digraph) $\mathcal{D}=(\mathcal{V}, \mathcal{E})$, in which $\mathcal{V}$ denotes a set of vertices and $\mathcal{E}$ represents a set of edges, such that, an edge $\left(v_{j}, v_{i}\right)$ is directed from vertex $v_{j}$ to vertex $v_{i}$. Denote by $\mathcal{X}=\left\{x_{1}, \cdots, x_{n}\right\}$ and $\mathcal{Y}=\left\{y_{1}, \cdots, y_{p}\right\}$ the set of state vertices and output vertices, respectively. Denote by $\mathcal{E}_{\mathcal{X}, \mathcal{X}}=$ $\left\{\left(x_{i}, x_{j}\right): \bar{A}_{j i} \neq 0\right\}$ and $\mathcal{E}_{\mathcal{X}, \mathcal{Y}}=\left\{\left(x_{i}, y_{j}\right): \bar{C}_{j i} \neq 0\right\}$, and consider $\mathcal{D}(\bar{A})=\left(\mathcal{X}, \mathcal{E}_{\mathcal{X}, \mathcal{X}}\right)$ and $\mathcal{D}(\bar{A}, \bar{C})=\left(\mathcal{X} \cup \mathcal{Y}, \mathcal{E}_{\mathcal{X}, \mathcal{X}} \cup\right.$ $\left.\mathcal{E}_{\mathcal{X}, \mathcal{Y}}\right)$ to be the state digraph and system digraph, respectively.

A sequence of edges $\mathcal{P}=\left\{\left(v_{1}, v_{2}\right),\left(v_{2}, v_{3}\right)\right.$, $\left.\ldots,\left(v_{k-1}, v_{k}\right)\right\}$, with $k \geq 2$, is called $a$ path from $v_{1}$ to $v_{k}$ and in short denoted by $v_{1} \rightsquigarrow v_{k}$. Notice that the vertices do not have to be necessarily distinct. The length of the path $\mathcal{P}$ is given by the number of edges it contains. If the length of the path $\mathcal{P}$ is $p$ then we denote it by $v_{1} \stackrel{p}{\rightsquigarrow} v_{k}$.

For any vertex $x_{i} \in \mathcal{X}$, we can define the out-neighboring set $\mathcal{N}^{+}\left(x_{i}\right)=\left\{x_{j} \in \mathcal{X}: \quad\left(x_{i}, x_{j}\right) \in \mathcal{E}_{\mathcal{X}, \mathcal{X}}\right\} \cup\left\{y_{j} \in \mathcal{Y}:\right.$ $\left(x_{i}, y_{j}\right) \in \mathcal{E}_{\mathcal{X}, \mathcal{Y}\}}$, and we can extend the definition to an arbitrary set $\mathcal{X}_{s} \subseteq \mathcal{X}$ as follows:

$$
\mathcal{N}^{+}\left(\mathcal{X}_{s}\right)=\bigcup_{x_{i} \in \mathcal{X}_{s}} \mathcal{N}^{+}\left(x_{i}\right) .
$$

We say that digraph $\mathcal{D}(\bar{A}, \bar{C})$ has a contraction if there exists $\mathcal{X}_{s} \subseteq \mathcal{X}$ such that $\left|\mathcal{N}^{+}\left(\mathcal{X}_{s}\right)\right|<\left|\mathcal{X}_{s}\right|$. Otherwise, we say that $\mathcal{D}(\bar{A}, \bar{C})$ is free of contractions. Subsequently, we have the following characterization of the structurally observable pairs.

Theorem 1 ([3]): Let $\bar{A} \in\{0,1\}^{n \times n}, \bar{C} \in\{0,1\}^{p \times n}$ and $\mathcal{D}(\bar{A}, \bar{C})=\left(\mathcal{X} \cup \mathcal{Y}, \mathcal{E}_{\mathcal{X}, \mathcal{X}} \cup \mathcal{E}_{\mathcal{X}, \mathcal{Y}}\right)$ be the system digraph. The pair $(\bar{A}, \bar{C})$ is structurally observable if and only if both conditions hold:

(i) the digraph $\mathcal{D}(\bar{A}, \bar{C})$ is free of contractions; 
(ii) for every $x_{i} \in \mathcal{X}$ there exists $y_{j} \in \mathcal{Y}$ in $\mathcal{D}(\bar{A}, \bar{C})$ such that $x_{i} \rightsquigarrow y_{j}$.

Before proceeding to the characterization of the structural observability index, we introduce the following two definitions.

Definition 1 (Output-Connected Set): Let $\mathcal{D}(\bar{A}, \bar{C})=(\mathcal{X} \cup$ $\left.\mathcal{Y}, \mathcal{E}_{\mathcal{X}, \mathcal{X}}, \mathcal{E}_{\mathcal{X}, \mathcal{Y}}\right)$, where $\mathcal{X}=\left\{x_{1}, x_{2}, \ldots, x_{n}\right\}$ and $\mathcal{Y}=$ $\left\{y_{1}, y_{2}, \ldots, y_{p}\right\}$, be the system digraph associated with some structural pair $(\bar{A}, \bar{C})$. The set $\mathcal{Y}_{j}^{k}$ associated with $y_{j}$, with $j \in\{1, \ldots, p\}$ and $k \in\{1, \ldots, n\}$, defined as $\mathcal{Y}_{j}^{k}=\left\{x_{i} \in\right.$ $\left.\mathcal{X}: x_{i} \stackrel{k}{\rightsquigarrow} y_{j}\right\}$ is called the output-connected set of $y_{j}$ at iteration $k$.

Definition 1 is suitable for recursive computations: if we consider that the output variable $y_{j}$ is assigned to the state variable $x_{i}$, i.e., $x_{i} \stackrel{1}{\rightsquigarrow} y_{j}$, then $\mathcal{Y}_{j}^{1}=\left\{x_{i}\right\}$ and

$\mathcal{Y}_{j}^{k}=\left\{x_{k} \in \mathcal{X}:\left(x_{k}, x_{l}\right) \in \mathcal{E}_{\mathcal{X}, \mathcal{X}}\right.$ with $\left.x_{l} \in \mathcal{O}_{j}^{k-1}\right\}$ for $k \geq 2$.

Definition 2 (Partial Transversal): Let $S$ be a finite set and let $\mathcal{S}=\left\{S_{1}, S_{2}, \ldots, S_{n}\right\}$ be a finite collection of subsets of $S$, that is, $S_{i} \subseteq S$ for each $i \in\{1,2, \ldots, n\}$. A partial transversal with respect to $\mathcal{S}$ consists of a set $\mathcal{A} \subset S$ whose elements are in a bijective relation with a sub-collection of $\mathcal{S}$.

Now, we can present the characterization of pairs $(\bar{A}, \bar{C})$ with a specified structural observability index.

Theorem 2 ([14]): Let $\mathcal{D}(\bar{A}, \bar{C})=\left(\mathcal{X} \cup \mathcal{Y}, \mathcal{E}_{\mathcal{X}, \mathcal{X}} \cup \mathcal{E}_{\mathcal{X}, \mathcal{Y}}\right)$, where $\mathcal{X}=\left\{x_{1}, x_{2}, \ldots, x_{n}\right\}$ and $\mathcal{Y}=\left\{y_{1}, y_{2}, \ldots, y_{p}\right\}$, be the system digraph associated with the pair $(\bar{A}, \bar{C})$. The structural pair $(\bar{A}, \bar{C})$ has structural observability index $\mu_{G}(\bar{A}, \bar{C}) \leq L$, $L \in\{1, \ldots, n\}$, if and only if the following conditions hold

(i) the system digraph $\mathcal{D}(\bar{A}, \bar{C})$ is free of contractions;

(ii) $\mathcal{X}$ is partial transversal with respect to the collection of the output-connected sets $\left\{\mathcal{Y}_{j}^{k}\right\}_{1 \leq j \leq p, 1 \leq k \leq L}$.

Consequently, we have the following result.

Corollary 1 ([14]): Let $\mathcal{D}(\bar{A}, \bar{C})=\left(\mathcal{X} \cup \mathcal{Y}, \mathcal{E}_{\mathcal{X}, \mathcal{X}}, \mathcal{E}_{\mathcal{X}, \mathcal{Y}}\right)$, where $\mathcal{X}=\left\{x_{1}, x_{2}, \ldots, x_{n}\right\}$ and $\mathcal{Y}=\left\{y_{1}, y_{2}, \ldots, y_{p}\right\}$, be the system digraph associated with the pair $(\bar{A}, \bar{C})$. If the pair $(\bar{A}, \bar{C})$ has structural observability index $\mu_{G}(\bar{A}, \bar{C})$, then for each state variable $x_{i} \in \mathcal{X}$ there exists a directed path to some $y_{j} \in \mathcal{Y}$ with length less than or equal to $\mu_{G}(\bar{A}, \bar{C})$.

\section{Problem Statement}

Let the power electrical grid be given by a discrete-time dynamics, linearized under normal operating conditions, whose structure is given by $\bar{A} \in\{0,1\}^{n \times n}$. In this paper we aim to address the following two problems:

$\mathcal{P}_{1}$ Given $\bar{A}$ and $\mu \in\{1, \ldots, n\}$, determine $\mathcal{J}^{*}$ such that

$$
\begin{array}{r}
\mathcal{J}^{*}=\arg \min _{\mathcal{J} \subset\{1, \ldots, n\}}|\mathcal{J}| \\
\text { s.t. } \quad \mu_{G}\left(\bar{A}, \square_{n}^{\mathcal{J}}\right) \leq \mu,
\end{array}
$$

where $\square_{n}^{\mathcal{J}}$ denotes the sub matrix (of the identity matrix) formed by the rows with indices in $\mathcal{J}$.
$\mathcal{P}_{2}$ Given $\bar{A}$ and the number of dedicated ${ }^{1}$ sensors $p \in$ $\{1, \ldots, n\}$, determine the minimal attainable structural observability index $\mu^{*}$, i.e.,

$$
\begin{array}{cc}
\mu^{*}=\arg \min _{\mu \in\{1, \ldots, n\}} & \mu \\
\text { s.t. } \quad \mu_{G}\left(\bar{A}, \square_{n}^{\mathcal{J}}\right) \leq \mu \\
& |\mathcal{J}| \leq p, \mathcal{J} \subset\{1, \ldots, n\} .
\end{array}
$$

\section{Main Results}

In this section, we present the main results of this paper that are as follows: first, we provide some preliminary results that sharpen the intuition about the conditions required to obtain a solution to $\mathcal{P}_{1}$, which is generally NP-hard. Then, we provide a greedy algorithm (Algorithm 1) that approximates the solution to $\mathcal{P}_{1}$ efficiently (i.e., polynomially in the size of the state space) and with some optimality guarantees, see Theorem 3 and Theorem 4. Finally, we propose an algorithm (Algorithm 2) that solves $\mathcal{P}_{2}$ by resorting to Algorithm 1.

We start by introducing a result on the necessary conditions for the solutions to $\mathcal{P}_{1}$, that can be obtained as consequences of Corollary 1.

Corollary 2: Consider $\mathcal{P}_{1}$ with $\bar{A} \in\{0,1\}^{n \times n}$ and desired structural observability index $\mu$ as input. If $\mathcal{J}$ is a solution of $\mathcal{P}_{1}$, then

$$
|\mathcal{J}| \geq\left\lceil\frac{n}{\mu}\right\rceil
$$

Before presenting a strategy to address $\mathcal{P}_{1}$, let us analyze two simple cases. The first occurs when $\mu=1$, for which a necessary (and sufficient) condition, by Corollary 2 , is $|\mathcal{J}| \geq\left\lceil\frac{n}{\mu}\right]=n$; thus, $\mathcal{J}=\{1,2, \ldots, n\}$, i.e., a dedicated output must be assigned to each state variable of the dynamical system (regardless of its dynamic structure). On the other hand, if we only aim to ensure that the system is structurally observable, i.e., $\mu \leq n$, then an efficient (polynomial in the size of the state space) can also be obtained as provided in [8].

Because the problem $\mathcal{P}_{1}$ is generally NP-hard [14], efficient algorithms to determine a solution are unlikely to exist. Therefore, hereafter we propose an approximation scheme that relies on greedy strategies and using techniques based on submodular function optmization [1], we derive estimates on the optimality gap of the solutions obtained.

By comparing Theorem 1 with Theorem 2, we can see that the difference lies on condition (ii). If we consider structural digraphs $\mathcal{D}(\bar{A})$ with a self-loop on each variable, we can conclude that regardless of the output matrix considered, the resulting system $\mathcal{D}(\bar{A}, \bar{C})$ is contraction-free. Therefore, in that case, the only condition that needs to be verified is condition (ii). That is, there must exist a partial transversal on the output-connected sets (Definition 2) such that the union equals the whole state vertex set. Thus, the hardness

\footnotetext{
${ }^{1} \mathrm{By}$ dedicated we mean that the sensor is connected to a single state variable, or equivalently, the corresponding row in the output matrix is canonical (i.e., consists of a single non-zero entry, the remaining $n-1$ entries being zero).
} 
of $\mathcal{P}_{1}$ comes from that condition. In an attempt to find an approximate solution, we will consider Algorithm 1, where we assume that in the digraph $\mathcal{D}(\bar{A})$ there exists a self-loop on each variable, i.e., $\bar{A}$ has non-zero diagonal entries.

ALGORITHM 1: Find an approximate solution to $\mathcal{P}_{1}$ when $\mathcal{D}(\bar{A})$ has self-loops on all state variables

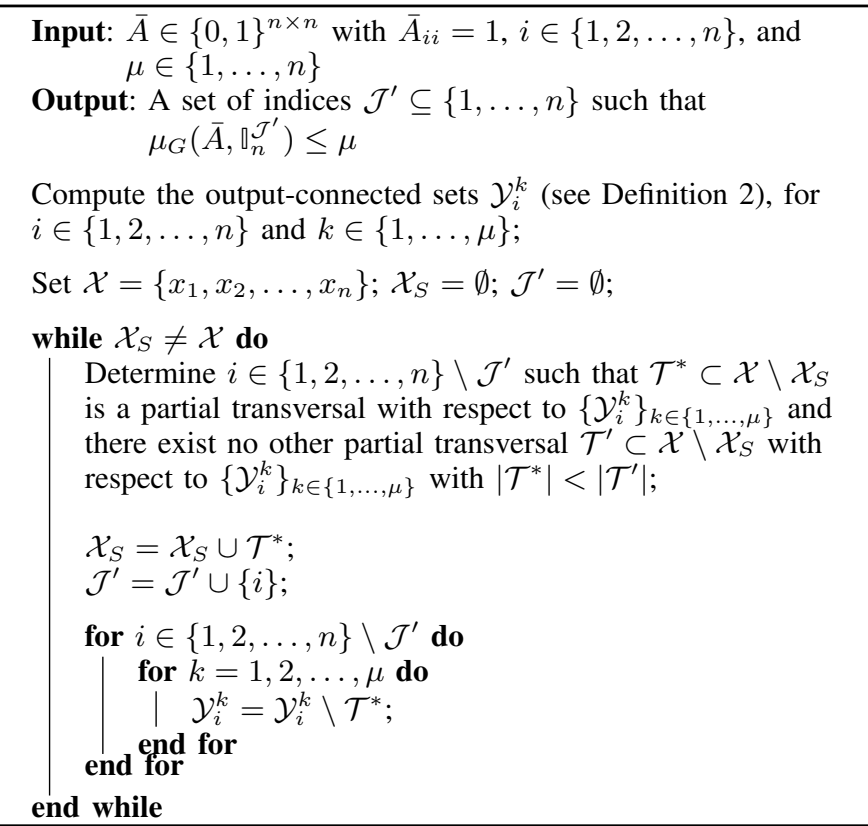

First, we show that the solution obtained by Algorithm 1 is feasible.

Theorem 3: The set $\mathcal{J}^{\prime} \subset\{1, \ldots, n\}$ obtained using Algorithm 1 is such that $\mu_{G}\left(\bar{A}, \square_{n}^{\mathcal{J}^{\prime}}\right) \leq \mu$.

Proof: First, notice that Algorithm 1 always stops, since in the worst case scenario $\mathcal{J}^{\prime}=\{1, \ldots, n\}$ and there exists a partial transversal in bijective relation with $\mathcal{Y}_{i}^{1}$ for $i \in \mathcal{J}^{\prime}$. Further, when the algorithm stops we have a partial transversal on the output-connected sets $\left\{\mathcal{Y}_{i}^{k}\right\}_{i \in \mathcal{J}^{\prime}, 1 \leq k \leq \mu}$, that equals the state vertex set. Since we are considering that the directed graph $\mathcal{D}(\bar{A})$ has a self-loop on each state variable, then $\mathcal{D}\left(\bar{A}, \square_{n}^{\mathcal{J}}\right)$ is free of contractions. Therefore, since conditions (i)-(ii) of Theorem 2 hold, the pair $\left(\bar{A}, \square_{n}^{\mathcal{J}}\right)$ has a structural observability index $\mu_{G}\left(\bar{A}, \square_{n}^{\mathcal{J}}\right) \leq \mu$.

In fact, Algorithm 1 consists in a polynomial (in the size of the state space) greedy strategy that ensures the following optimality guarantees.

Theorem 4: Algorithm 1 runs in polynomial time and is $\left(1-\frac{1}{e}\right)$-suboptimal, i.e., the solution obtained is at most $\frac{1}{e}$ worst than the optimal solution.

Proof: [SKETCH] It is easy to see that the Algorithm 1 runs in polynomial time since in the worst case scenario the algorithm considers $\mathcal{J}=\{1, \ldots, n\}$, which implies that $\mathcal{X}_{S}=\mathcal{X}$, i.e., it stops. Next, we notice that $-\mu\left(\bar{A}, \square_{n}^{\mathcal{J}}\right)$ is a sub-modular function [1], i.e., if $\mathcal{J}^{\prime} \subset \mathcal{J} \subset\{1, \ldots, n\}$ and $j \in\{1, \ldots, n\} \backslash \mathcal{J}$, then $-\mu\left(\bar{A}, \square_{n}^{\mathcal{J}^{\prime} \cup\{j\}}\right)-\mu\left(\bar{A}, \square_{n}^{\mathcal{J}^{\prime}}\right) \geq$
$-\mu\left(\bar{A}, \square_{n}^{\mathcal{J} \cup\{j\}}\right)-\mu\left(\bar{A}, \square_{n}^{\mathcal{J}}\right)$. To verify this property, notice that by definition of structural observabiltiy index, if $\mu\left(\bar{A}, \square_{n}^{\mathcal{J}}\right)=$ $\infty$ the system is not structurally observable, hence, the inequality immediately holds. Alternatively, if $\mu\left(\bar{A}, \square_{n}^{\mathcal{J}}\right)<\infty$, then the inequality follows by recalling the monoticity of the structural observability index, as already pointed out in Corollary 1 and Corollary 2 . Given that $-\mu\left(\bar{A}, \square_{n}^{\mathcal{J}}\right)$ is sub-modular, the proposed greedy algorithm follows those steps commonly used to maximize such functions (in other words, minimizing the structural observability index), see [1] for details. These greedy algorithms ensure that the solution obtained is $\left(1-\frac{1}{e}\right)$ suboptimal, and the result follows.

From Theorem 4, we have that the size of $\mathcal{J}^{\prime}$ obtained using Algorithm 1 has at most approximately $37 \%$ more indices than the optimal solution $\mathcal{J}^{*}$ to $\mathcal{P}_{1}$, which we recall to be in general NP-hard to determine.

We can address $\mathcal{P}_{2}$ by solving instances of $\mathcal{P}_{1}$ as proposed in Algorithm 2. Therefore, a solution to $\mathcal{P}_{2}$ can be approximated by resorting to Algorithm 1, when approximating the solution to $\mathcal{P}_{1}$ in Algorithm 2.

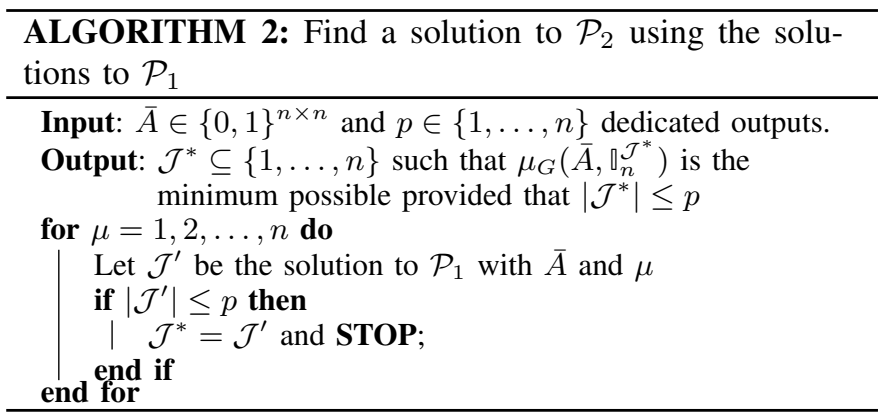

In the next section, we provide a description of a linearized power electric grid model (under normal operation conditions), through which we illustrate the performance of the proposed approximations schemes to address $\mathcal{P}_{1}$ and $\mathcal{P}_{2}$.

\section{Linearized Model of Dynamic Power Electric GRID}

The electric power grid considered for illustrative purposes in the present paper is modeled as in [5] and consists in a linearized model under normal operating conditions. It can be written in terms of interconnected dynamical subsystems that consist of generators and loads denoted by $G_{i}$ and $L_{j}$. The state digraph representation of the generators and loads is given by $\mathcal{D}\left(\bar{A}_{G_{i}}\right)$ and $\mathcal{D}\left(\bar{A}_{L_{j}}\right)$ as depicted in Figure 1(a). The variable $P_{T_{G_{i}}}$ represents the mechanical power of the turbine of the generator $G_{i}, \omega_{G_{i}}$ the generator $G_{i}$ frequency, $a_{G_{i}}$ the valve opening of the load $L_{j}, l_{L_{j}}$ the real energy consumed by the load between two samples $(k$ and $(k+1))$ of the load $L_{j}$ and $\omega_{L_{j}}$ the frequency at the load $L_{j}$ location.

In addition, the different components are connected through the injected and received power from the network at the connection site, whose dynamics depends of the frequency of the components directly on the adjacency buses. More precisely, additional variables, $P_{G_{i}}$ and $P_{L_{j}}$, need to be considered for each generator and load, respectively, and 
the digraph representation of the dynamics has bidirectional connection between these new variables and the frequency of the corresponding components, see Figure 1(a). Further, if two buses are connected through a transmission line or two dynamic components are in the same bus, then the frequency of the component $i$ affects the dynamics of the power injection in the network at site $j$ and vice-versa; hence, the digraph of the interconnected dynamics has outgoing edges from the frequencies of the components into the power injections of the adjacent sites, see, for instance, Figure 1(a), where a generator is connected to bus $i$, a load is connected to bus $j$ and these two buses are connected through a transmission line, see [5] for details.

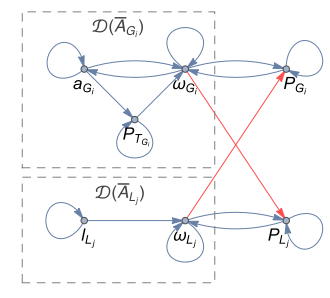

(a)

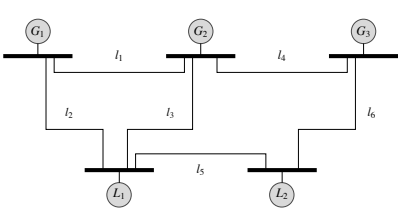

(b)
Fig. 1. (a) The generator's state digraph $\mathcal{D}\left(\bar{A}_{G_{i}}\right)$ and the load's state digraph $\mathcal{D}\left(\bar{A}_{L_{i}}\right)$ contains the state variables described in Section V. The generators and loads are coupled in the network through the power injected and received by the different components, and the power depends upon the frequency of the neighboring components (or adjacent buses), i.e., the buses containing the dynamic components are connected by transmission lines, as depicted by the red edges in the case where generator $G_{i}$ and load $L_{j}$ are in the same bus or adjacent buses; (b) The IEEE 5-bus testbed system.

\section{ILLUSTRATIVE EXAMPLE}

Hereafter, we illustrate how to provide an approximate solution to $\mathcal{P}_{1}$ given the linear-time invariant system that models the IEEE 5-bus testbed system depicted in Figure 1(b) as described in Section V. The collection of state variables to which the dedicated outputs need to be assigned to in order to obtain a certain observability index is given as follows:

\begin{tabular}{|c|c|c|}
\hline$\mu$ & 6 & 5 \\
\hline $\mathcal{J}^{\prime}$ & $\left\{P_{G_{1}}, P_{L_{1}}, P_{L_{2}}, P_{G_{3}}\right\}$ & $\left\{P_{G_{1}}, P_{L_{1}}, P_{L_{2}}, P_{G_{3}}\right\}$ \\
\hline \hline$\mu$ & 4 & 3 \\
\hline $\mathcal{J}^{\prime}$ & $\left\{\omega_{G_{1}}, \omega_{G_{2}}, \omega_{G_{3}}, P_{L_{1}}, P_{L_{2}}\right\}$ & $\left\{\omega_{G_{1}}, P_{G_{1}}, \omega_{G_{2}}, P_{G_{2}}\right.$, \\
& & $\left.\omega_{G_{3}}, P_{G_{3}}, P_{L_{1}}, P_{L_{2}}\right\}$ \\
\hline
\end{tabular}

Notice that the same subset of state variables was obtained to ensure a structural observability index $\mu=5$ and $\mu=6$, and, as expected, a smaller $\mu$ requires a larger subset of state variables that need to be measured by dedicated outputs. Now, suppose that we were told that we could use at most four dedicated outputs, and we wanted to minimize the structural observability index, i.e., we were interested in solving $\mathcal{P}_{2}$, then by resorting to Algorithm 2 (and checking the above table) we conclude that the best (approximate) assignment of those outputs would be $\left\{P_{G_{1}}, P_{L_{1}}, P_{L_{2}}, P_{G_{3}}\right\}$ incurring in a structural observability index of $\mu=5$.

\section{CONCLUSIONS AND FURTHER RESEARCH}

In this paper, we have provided a suboptimal algorithm to determine the minimal number and placement of dedicated sensors that achieves a desired structural observability index.
We also provided an algorithm to, given the number of sensors to be deployed, obtaining the placement that ensures that the structural observability index is minimal.

Further research may consist of determining lower and tighter upper bounds for the approximation algorithms provided. This may be accomplished by considering the specific structure of the system dynamics and the output-connected sets. Further, one may be able to obtain optimal algorithms for certain more restricted classes of state digraph structures.

\section{REFERENCES}

[1] F. Bach. Learning with Submodular Functions: A Convex Optimization Perspective. ArXiv e-prints, November 2011.

[2] C T Chen. Linear System Theory and Design. Holt, Rinehart, and Winston, 1984.

[3] J.-M. Dion, C. Commault, and J. Van Der Woude. Survey generic properties and control of linear structured systems: a survey. Automatica, 39(7):1125-1144, July 2003.

[4] Y.-F. Huang, S. Werner, J. Huang, N. Kashyap, and V. Gupta. State estimation in electric power grids: Meeting new challenges presented by the requirements of the future grid. Signal Processing Magazine, IEEE, 29(5):33-43, 2012.

[5] M. D. Ilić, L. Xie, U. A. Khan, and J. M. F. Moura. Modeling of future cyber-physical energy systems for distributed sensing and control. IEEE Transactions on Systems, Man and Cybernetics, Part A: Systems and Humans, 40(4):825-838, July 2010.

[6] H. Mortazavian. On $k$-controllability and $k$-observability of linear systems. In Analysis and Optimization of Systems, pages 600-612. Springer, 1982.

[7] F. Pasqualetti, S. Zampieri, and F. Bullo. Controllability metrics, limitations and algorithms for complex networks. IEEE Transactions on Control of Network Systems, 1(1):40-52, 2014.

[8] S. Pequito, S. Kar, and A. P. Aguiar. A framework for structural input/output and control configuration selection of large-scale systems. Submitted to IEEE Transactions on Automatic Control, 2013.

[9] S. Pequito, S. Kar, and A. P. Aguiar. A structured systems approach for optimal actuator-sensor placement in linear time-invariant systems. Proceedings of American Control Conference, 2013.

[10] S. Pequito, Q. Liu, S. Kar, and M. D. Ilic. PMU placement to ensure observable frequency and voltage dynamics: A structured system approach. 2014 47th Hawaii International Conference on System Sciences, 0:2327-2336, 2013.

[11] A. G. Phadke, J. S. Thorp, R. F. Nuqui, and M. Zhou. Recent developments in state estimation with phasor measurements. In Power Systems Conference and Exposition, 2009. PSCE '09. IEEE/PES, pages $1-7,2009$.

[12] J. Qi, K. Sun, and W. Kang. Optimal PMU placement for power system dynamic state estimation by using empirical observability Grammian, journal $=$ ArXiv e-prints, archiveprefix $=$.

[13] K. J. Reinschke. Multivariable control: a graph theoretic approach, volume 108 of Lect. Notes in Control and Information Sciences. SpringerVerlag, 1988.

[14] P. Rocha. Output selection for large scale structural systems: a matroid theory approach. MSc thesis, Department of Electrical and Computer Engineering, Faculty of Engineering, University of Porto, Porto, Portugal, July 2014.

[15] C. Sueur and G. Dauphin-Tanguy. Controllability indices for structured systems. Linear algebra and its applications, 250:275-287, 1997.

[16] T. H. Summers, F. L. Cortesi, and J. Lygeros. On Submodularity and Controllability in Complex Dynamical Networks. ArXiv e-prints, April 2014.

[17] S. Sundaram and C. N. Hadjicostis. Structural controllability and observability of linear systems over finite fields with applications to multiagent systems. IEEE Transactions on Automatic Control, 58(1):60-73, 2013.

[18] V. Tzoumas, M. A. Rahimian, G. J. Pappas, and A. Jadbabaie. Minimal actuator placement with bounds on control effort, http://arxiv.org/abs/1409.3289. ArXiv, 2014.

[19] M. Zhao and A. Abur. Multi-area state estimation using synchronized phasor measurements. IEEE Transactions on Power Systems, 20(2):611617, 2005. 